\title{
Pedunculated cavernous hemangioma mimicking inflammatory fibroid polyp in a patient with ulcerative colitis
}

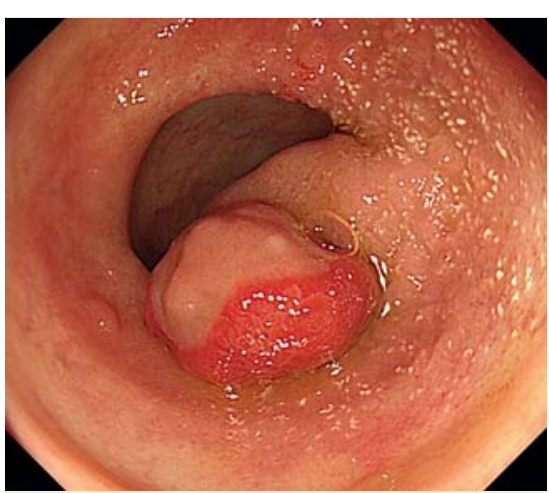

Fig. 1 Endoscopic view showing a pedunculated polyp, $10 \mathrm{~mm}$ in size, which exhibited superficial areas of ulceration on its surface and was located at the sigmoid colon.

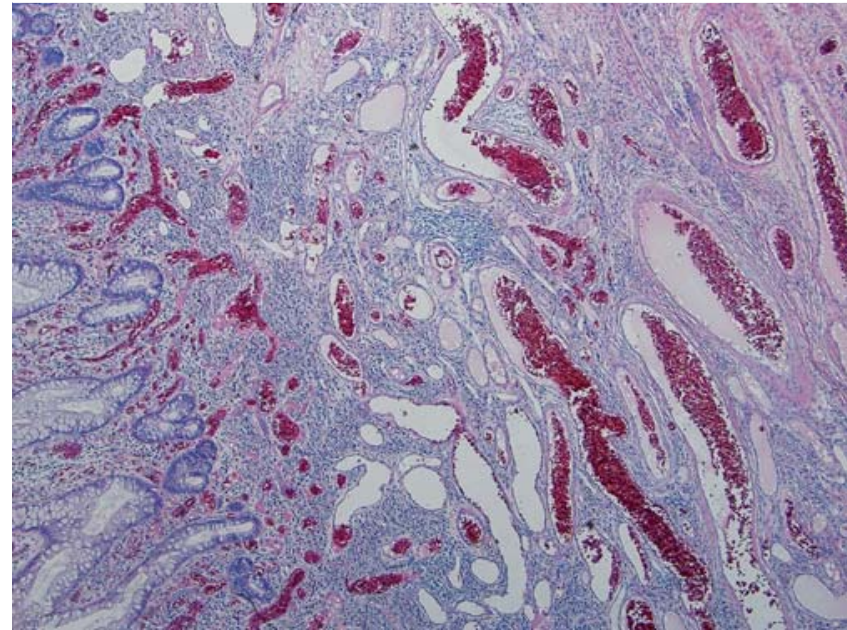

Fig. 2 Histologic examination revealed proliferation of dilated blood vessels and infiltration of mononuclear cells but no spindle cells positive for CD34.
A-29-year-old man with steroid-dependent ulcerative colitis had been treated with tacrolimus therapy. He underwent colonoscopy to evaluate the therapeutic effect of the tacrolimus therapy. Endoscopic examination revealed complete mucosal healing throughout the entire colon. However, a pedunculated polyp, $10 \mathrm{~mm}$ in size, which exhibited superficial areas of ulceration on its surface, was detected at the sigmoid colon ( $\bullet$ Fig. 1). This endoscopic finding was suggestive of inflammatory fibroid polyp (IFP). We considered this polyp to be the source of hematochezia and performed snare polypectomy. Histologic examination revealed a polyp with erosion and granulation at the surface and, in the submucosal area, proliferation of dilated blood vessels and infiltration of mononuclear cells ( $\mathbf{F i g . 2}$ ). Spindle cells positive for CD34 were not observed. This polyp was diagnosed as a pedunculated cavernous hemangioma.

Colonic hemangioma is a rare, nonmalignant lesion arising from the submucosal vascular plexuses and usually localized at the rectum and sigmoid colon. Characteristic endoscopic findings of colonic hemangioma are soft, dilated, easily collapsible, submucosal masses, ranging in color from deep wine to plum [1]. Of note, the endoscopic finding of this polyp mimicked that of IFP. In addition, the simultaneous occurrence of inflammatory bowel disease with IFP was reported. Thus, we first diagnosed this pedunculated polyp as IFP. However, histologic examination revealed prominent proliferation of dilated vessels, which was compatible with the histologic finding of colonic hemangioma. Colonic hemangioma occurring in association with ulcerative colitis is unknown, but our case may support the hypothesis that mucosal inflammation and intralesional microhemorrhage enhanced by the conjunction of an underlying ulcerative colitis contributes to the development of coincidental cavernous hemangioma [2]. Surgical treatment is the first choice for large or diffuse lesions of colonic hemangioma. In cases of the pedunculated colonic hemangioma, as in our case, the less invasive endoscopic resection may be preferable to surgery if possible [3].

\section{Competing interests: None}

Endoscopy_UCTN_Code_CCL_1AD_2AC

\section{K. Ueda , H. Nakase, H. Yaku, Y. Honzawa, T. Chiba}

Department of Gastroenterology and Hepatology, Graduate School of Medicine, Kyoto University, Kyoto, Japan

\section{References}

1 Vilallonga R, Espin Basany E, Armengol M. Cavernous hemangioma: unusual tumor of the transverse colon. Turk J Gastroenterol 2009; 20: 146-149

2 Erkan G, Alagõzlü H, Memiş L et al. Rapidly growing giant solitary cavernous hemangioma in a patient with ulcerative colitis. Turk J Gastroenterol 2008; 19: 271-275

3 van Deursen CT, Bujis J, Nap M. An uncommon polyp in the colon: a pedunculated cavernous hemangioma. Endoscopy 2008; 40 (Suppl 2): E127

Bibliography

DOI $10.1055 / \mathrm{s}-0029-1244144$

Endoscopy 2010; 42: E162

(c) Georg Thieme Verlag KG Stuttgart · New York . ISSN 0013-726X

\section{Corresponding author \\ H. Nakase, MD, PhD}

Department of Gastroenterology and Hepatology Endoscopic Medicine Kyoto University Hospital 54 Shogoin Kawahara-cho Sakyo-ku Kyoto, 606-8507

Japan

Fax: +81-75-7514303

hiropy@kuhp.kyoto-u.ac.jp 